\title{
Restaging after chemoradiotherapy for locally advanced esophageal cancer
}

\author{
Ingmar L. Defize ${ }^{1,2}$, Richard van Hillegersberg ${ }^{2}$, Stella Mook ${ }^{1}$, Gert J. Meijer ${ }^{1}$, Steven H. Lin ${ }^{3}$, \\ Jelle P. Ruurda ${ }^{2}$, Peter S. N. van Rossum ${ }^{1}$
}

${ }^{1}$ Department of Radiation Oncology, ${ }^{2}$ Department of Surgery, University Medical Center Utrecht, Utrecht, The Netherlands; ${ }^{3}$ Department of Radiation Oncology, The University of Texas MD Anderson Cancer Center, Houston, TX, USA

Correspondence to: Peter S. N. van Rossum, MD, PhD. Department of Radiation Oncology, University Medical Center Utrecht, Heidelberglaan 100, 3584CX Utrecht, The Netherlands. Email: P.S.N.vanRossum-2@umcutrecht.nl.

Provenance: This is an invited article commissioned by the Section Editor Dr. Dong Tian (Department of Thoracic Surgery, The Affiliated Hospital of North Sichuan Medical College, Nanchong, China).

Comment on: de Gouw DJJM, Klarenbeek BR, Driessen M, et al. Detecting pathological complete response in esophageal cancer after neoadjuvant therapy based on imaging techniques: a diagnostic systematic review and meta-analysis. J Thorac Oncol 2019;14:1156-71.

Submitted Oct 30, 2019. Accepted for publication Nov 11, 2019.

doi: $10.21037 / \mathrm{atm} .2019 .11 .57$

View this article at: http://dx.doi.org/10.21037/atm.2019.11.57

Restaging after chemoradiotherapy (CRT) for locally advanced esophageal cancer is a pivotal part of modern treatment individualization. de Gouw et al. performed a systematic review and meta-analysis determining the value of different modalities for the locoregional part of restaging, involving response assessment of the primary tumor and regional lymph nodes (1). Detection of potentially emerged distant metastasis is the other crucial part of restaging after CRT. The results of combined locoregional and distant restaging after CRT divides esophageal cancer patients into three groups, i.e., patients with (I) no evidence of residual locoregional disease nor distant spread, who might benefit from an active surveillance approach; or (II) persistent locoregional disease with no evidence of distant spread, who require subsequent surgery; or (III) either persistent or no residual locoregional disease with evidence of distant spread, who should be offered systemic treatment, mostly with palliative intent.

For the locoregional disease status after CRT, the diagnostic accuracy of restaging imaging modalities for detecting pathologic complete response (ypCR) was assessed by de Gouw et al. (1). The authors reviewed computed tomography (CT), ${ }^{18} \mathrm{~F}$-fluorodeoxyglucose positron emission tomography-CT $\left({ }^{18}\right.$ F-FDG PET-CT), endoscopic ultrasound (EUS), and magnetic resonance imaging (MRI) for this purpose. With pooled sensitivities for detecting ypCR of CT, PET-CT, EUS, and MRI of $35 \%, 62 \%, 1 \%$, and $80 \%$, respectively, and pooled specificities of $83 \%, 73 \%, 99 \%$, and $83 \%$, respectively, the authors concluded the modalities lack sufficient accuracy to identify complete responders independently. As such, the authors state that these modalities might be combined to achieve improved diagnostic performance. This touches upon an interesting and largely unexplored concept of a multimodality restaging approach, that was not yet further elaborated on. Also, as the main aim of de Gouw et al. was to identify locoregional pathologic complete responders, the authors did not comment on the restaging of distant disease. The overall value of restaging tools on a patient level (including both locoregional and distant assessment) deserves further discussion.

\section{Multimodal locoregional restaging}

The concept of multimodal locoregional restaging is based on the use of two or more complementary restaging modalities. The aim of a multimodal strategy is to yield the highest diagnostic performance with the least amount of modalities. Conventional modalities such as CT, ${ }^{18} \mathrm{~F}$-FDG PET-CT and EUS might play an important role, but modern methods such as diffusion-weighted MRI (DWMRI) are increasingly explored. The value of repeated DWMRI for locoregional response evaluation in esophageal cancer might have been underestimated in the review by de Gouw et al. (1). As the search by de Gouw et al. was 
updated to December 2017, more recent studies were missing, including a study by Fang et al., showing high predictive performance with a sensitivity and specificity of up to $100 \%$ for repeated DW-MRI $(1,2)$. Moreover, a recent meta-analysis by Cheng et al. including 7 DW-MRI studies reported superior DW-MRI results compared to the findings by de Gouw et al. with a pooled sensitivity and specificity of $93 \%$ and $85 \%$, respectively $(1,3)$.

For a near-future individualized treatment approach, we envision locoregional restaging will include repeated DWMRI because of a growing body of evidence on its superior performance, in combination with post-CRT endoscopic (bite-on-bite) biopsy because of its unbeatable positive predictive value for residual cancer (2-6). Besides repeated DW-MRI, the recent study of Fang et al. also found that changes in the total lesion glycolysis (TLG) during CRT on ${ }^{18} \mathrm{~F}$-FDG PET-CT were highly predictive [area under the curve (AUC): 0.95] for a ypCR (2). Therefore, ${ }^{18} \mathrm{~F}$-FDG PET-CT might be a valuable addition to the multimodal combination for locoregional restaging.

\section{Multimodal distant restaging}

As with locoregional restaging, a complementary multimodal restaging concept should be considered for the assessment of distant disease as well. Early distant recurrence is more prevalent and more deadly than locoregional disease recurrence after CRT and surgery for locally advanced esophageal cancer $(7,8)$. Even up to $20 \%$ of patients with a ypCR die within the first 2 years after therapy, mostly due to distant metastatic recurrence, underlining the crucial importance of accurate staging and restaging of distant spread. Initial staging ${ }^{18} \mathrm{~F}$-FDG PET is considered standard work-up as it provides complementary ability to detect (otherwise undetected) distant metastases in $5-28 \%$ of patients (9). In restaging after CRT, ${ }^{18} \mathrm{~F}-\mathrm{FDG}$ PET-CT correctly detects previously undetected distant (interval) metastases in $8 \%$ of patients (10). However, even in a comprehensive staging and restaging including repeated ${ }^{18}$ F-FDG PET-CT, (microscopic) distant spread before and/ or after CRT is still missed due to the implicit detection threshold of current diagnostic modalities.

In the meanwhile, improvements in genomic and molecular methods have led to a growing interest for using 'liquid biopsies', i.e., the analysis of tumor cells or tumor DNA obtained through the sampling of blood, in early cancer diagnosis, residual disease detection, and response monitoring (11). Circulating tumor DNA (ctDNA), for example, might provide a good reflection of the overall systemic tumor burden of a patient at initial presentation. Indeed, a ctDNA pilot study in esophageal cancer demonstrated a very strong association between post-CRT ctDNA presence and early distant recurrence (12). As such, we envision in the near future multimodal staging and restaging approaches may be enriched by liquid biopsies to more effectively stratify patients to adequate therapy.

\section{Envisioned risk-stratified management}

In Figure 1, we present an envisioned strategy for riskstratified management of patients with locally advanced esophageal cancer in the near future. The domain includes patients with locally advanced esophageal cancer who have no evidence of distant metastases at presentation, based on current standard diagnostic modalities including ${ }^{18} \mathrm{~F}$-FDG PET-CT. Baseline liquid biopsy is of envisioned value to detect a group of patients at high risk of (microscopic) distant spread, e.g., by demonstrating high ctDNA levels, which at that time point predominantly determines their prognosis. As such, these patients likely benefit most from upfront (induction) systemic therapy. Subsequent restaging (e.g., through repeated liquid biopsy) could then riskstratify these patients further to guide the focus of treatment to either the distant or locoregional disease status. In contrast, the prognosis of patients with an initial low risk of (microscopic) distant spread, e.g., with low or undetectable ctDNA levels, is mostly threatened by the locoregional disease at that point, and hence, these patients are eligible for commencement of CRT.

Although validation in large multicenter studies is awaited, DW-MRI early during CRT (e.g., 2-3 weeks after the start of CRT) has so far shown the highest potential to accurately predict pathologic response and is envisioned to play a role in stratifying good and poor locoregional responders. Potential value of ${ }^{18} \mathrm{~F}-\mathrm{FDG}$ PET-CT over DW-MRI alone early during CRT for locoregional response assessment is envisioned, and subject of validation in large studies currently as well. In good responders, after CRT restaging ${ }^{18} \mathrm{~F}$-FDG PET-CT and liquid biopsy could then stratify into 'undetectable disease' versus '(high risk of) distant disease' groups, eligible for active surveillance versus systemic treatment, respectively. In poor locoregional responders, distant restaging after CRT can stratify patients for systemic therapy in case of (high risk of) distant spread versus subsequent surgical treatment in case of a persisting low risk of (microscopic) distant spread. 


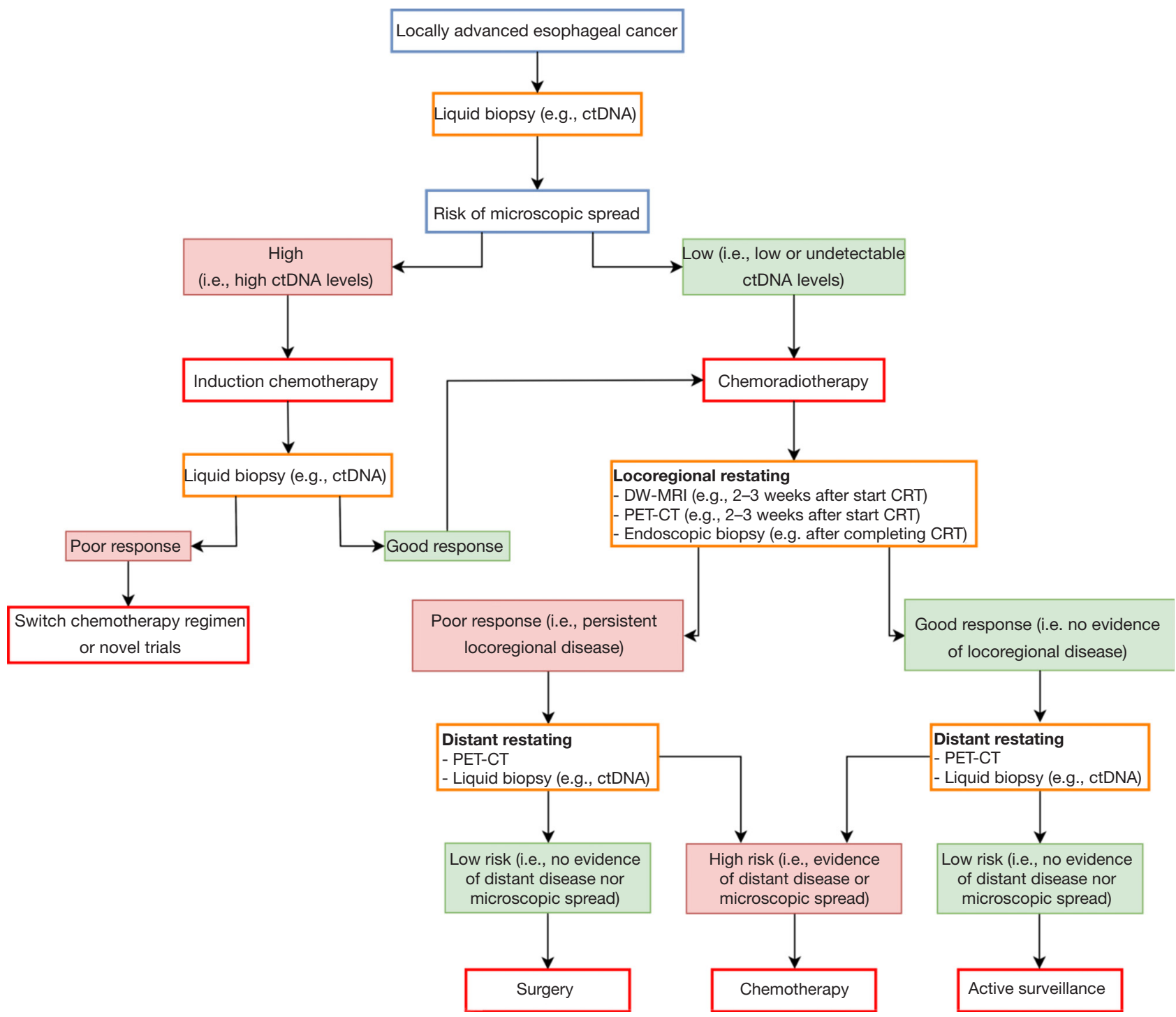

Figure 1 Envisioned strategy for risk-stratified management of patients with locally advanced esophageal cancer. CRT, chemoradiotherapy; ctDNA, circulating tumor DNA; DW-MRI, diffusion-weighted magnetic resonance imaging; PET-CT, positron emission tomographycomputed tomography.

\section{Ongoing trials}

Currently, several clinical trials are ongoing with the potential to complementarily provide the desired information that is needed to move the individualized approach from vision to reality. First, the appropriate combination of restaging modalities should be explored, meticulously reporting on the incremental value of the modalities (beyond each other) for locoregional as well as distant restaging. The optimal multimodal (re)staging approach is subject of investigation in 200 patients in the ongoing Dutch multicenter PRIDE trial incorporating repeated multimodal MRI, ${ }^{18}$ F-FDG PET-CT, EUS, endoscopic biopsy, and liquid biopsies, with pathology after surgery as reference standard (13). Besides knowledge on diagnostic performances, knowledge about potential unique risks associated with individualized approaches (e.g., active surveillance in patients with a clinical complete response, risk of missing locoregional or distant disease, consequences of delayed surgery) is essential. The SANO and ESOSTRATE trials will include 600 patients (300 within in each trial), and will provide extensive insights in 
the risks and consequences of active surveillance $(14,15)$.

Moving forward, the envisioned treatment approach carries the potential to enable the selection of treatment that is best for the individual patient at that time point, and omission of components that do not contribute to the well-being of the patient. Improving the quality of life and potentially the survival for many esophageal cancer patients makes this a goal highly worth pursuing.

\section{Acknowledgments}

None.

\section{Footnote}

Conflicts of Interest: The authors have no conflicts of interest to declare.

Ethical Statement: The authors are accountable for all aspects of the work in ensuring that questions related to the accuracy or integrity of any part of the work are appropriately investigated and resolved.

\section{References}

1. de Gouw DJJM, Klarenbeek BR, Driessen M, et al. Detecting pathological complete response in esophageal cancer after neoadjuvant therapy based on imaging techniques: a diagnostic systematic review and metaanalysis. J Thorac Oncol 2019;14:1156-71.

2. Fang P, Musall BC, Son JB, et al. Multimodal imaging of pathologic response to chemoradiation in esophageal cancer. Int J Radiat Oncol Biol Phys 2018;102:996-1001.

3. Cheng B, Yu J. Predictive value of diffusion-weighted MR imaging in early response to chemoradiotherapy of esophageal cancer: a meta-analysis. Dis Esophagus 2019. doi: 10.1093/dote/doy065.

4. van Rossum PS, van Lier AL, van Vulpen M, et al. Diffusionweighted magnetic resonance imaging for the prediction of pathologic response to neoadjuvant chemoradiotherapy in esophageal cancer. Radiother Oncol 2015;115:163-70.

5. van Rossum PSN, Goense L, Meziani J, et al. Endoscopic biopsy and EUS for the detection of pathologic complete response after neoadjuvant chemoradiotherapy in esophageal cancer: a systematic review and meta-analysis. Gastrointest Endosc 2016;83:866-79.

6. Noordman BJ, Spaander MCW, Valkema R, et al. Detection of residual disease after neoadjuvant chemoradiotherapy for oesophageal cancer (preSANO): a prospective multicentre, diagnostic cohort study. Lancet Oncol 2018;19:965-74.

7. Shapiro J, van Lanschot JJB, Hulshof MCCM, et al. Neoadjuvant chemoradiotherapy plus surgery versus surgery alone for oesophageal or junctional cancer (CROSS): long-term results of a randomised controlled trial. Lancet Oncol 2015;16:1090-8.

8. Parry K, Visser E, van Rossum PS, et al. Prognosis and treatment after diagnosis of recurrent esophageal carcinoma following esophagectomy with curative intent. Ann Surg Oncol 2015;22 Suppl 3:S1292-300.

9. van Rossum PSN, van Lier ALHMW, Lips IM, et al. Imaging of oesophageal cancer with FDG-PET/CT and MRI. Clin Radiol 2015;70:81-95.

10. Kroese TE, Goense L, van Hillegersberg R, et al. Detection of distant interval metastases after neoadjuvant therapy for esophageal cancer with 18F-FDG PET(/CT): a systematic review and meta-analysis. Dis Esophagus 2018. doi: 10.1093/dote/doy055.

11. Wan JCM, Massie C, Garcia-Corbacho J, et al. Liquid biopsies come of age: towards implementation of circulating tumour DNA. Nat Rev Cancer 2017;17:223-38.

12. Azad TD, Chaudhuri AA, Newman A, et al. Pretreatment circulating tumor DNA for risk stratification of locally advanced esophageal cancer treated with chemoradiation and surgery. Int J Radiat Oncol Biol Phys 2017;99:S90-1.

13. Borggreve AS, Mook S, Verheij M, et al. Preoperative image-guided identification of response to neoadjuvant chemoradiotherapy in esophageal cancer (PRIDE): a multicenter observational study. BMC Cancer 2018;18:1006.

14. Noordman BJ, Wijnhoven BPL, Lagarde SM, et al. Neoadjuvant chemoradiotherapy plus surgery versus active surveillance for oesophageal cancer: a stepped-wedge cluster randomised trial. BMC Cancer 2018;18:142.

15. Bedenne L, Mariette C. Comparison of systematic surgery versus surveillance and rescue surgery in operable oesophageal cancer with a complete clinical response to radiochemotherapy (Esostrate). 2015. Available online: https://clinicaltrials.gov/show/nct02551458

Cite this article as: Defize IL, van Hillegersberg R, Mook S, Meijer GJ, Lin SH, Ruurda JP, van Rossum PSN. Restaging after chemoradiotherapy for locally advanced esophageal cancer. Ann Transl Med 2019;7(Suppl 8):S288. doi: 10.21037/ atm.2019.11.57 